\title{
The Vibration Plethysmographic Method of Arterial Compliance Analysis in Dependence on Transmural Pressure
}

\author{
J. MOUDR ${ }^{1}$, J. SVAČINOVÁ ${ }^{1}$, E. ZÁVODNÁ ${ }^{2,1}$, N. HONZÍKOVÁ ${ }^{1}$ \\ ${ }^{1}$ Department of Physiology, Faculty of Medicine, Masaryk University, Brno, Czech Republic, \\ ${ }^{2}$ International Clinical Research Center, St. Anne's University Hospital Brno, Brno, Czech Republic
}

Received March 14, 2014

Accepted July 28, 2014

\section{Summary}

The aim of this study was to obtain a detailed analysis of the relationship between the finger arterial compliance $C[\mathrm{ml} / \mathrm{mm} \mathrm{Hg}]$ and the arterial transmural pressure $P_{t}[\mathrm{~mm} \mathrm{Hg}]$. We constructed a dynamic plethysmograph enabling us to set up a constant pressure $P_{c s s}[\mathrm{~mm} \mathrm{Hg}]$ and a superimposed fast pressure vibration in the finger cuff (equipped with a source of infra-red light and a photoelectric sensor for the measurement of arterial volume). $P_{\text {css }}$ could be set on the required time interval in steps ranging between 30 and $170 \mathrm{~mm} \mathrm{Hg}$, and on sinusoidal pressure oscillation with an amplitude $P_{c a}(2 \mathrm{~mm} \mathrm{Hg})$ and a frequency $f$ $(20,25,30,35,40 \mathrm{~Hz})$. At the same time continuous blood pressure BP was measured on the adjacent finger (Portapres). We described the volume dependence of a unitary arterial length on the time-varying transmural pressure acting on the arterial wall (externally $P_{c s s}+P_{c a} . \sin (2 n f)$, internally $B P$ ) by a second-order differential equation for volume. This equation was linearized within a small range of selected BP. In the next step, a Fourier transform was applied to obtain the frequency characteristic in analytic form of a complex linear combination of frequency functions. While series of oscillations $\left[P_{c a}, f\right]$ were applied for each $\mathrm{P}_{\mathrm{css}}$, the corresponding response of the plethysmogram was measured. Amplitude spectra were obtained to estimate coefficients of the frequency characteristic by regression analysis. We determined the absolute value: elastance $\mathrm{E}$, and its inverse value: compliance $(C=1 / E)$. Then, $C=C\left(P_{t}\right)$ was acquired by applying sequences of oscillations for different $P_{\text {css }}$ (and thus $P_{t}$ ) by the above-described procedure. This methodology will be used for the study of finger arterial compliance in different physiological and pathological conditions.

\section{Key words}

Arterial compliance • Finger arterial pressure • Plethysmography

- Transmural arterial pressure • Frequency characteristic

\section{Corresponding author}

J. Svacinova, Department of Physiology, Faculty of Medicine, Masaryk University, Kamenice 5, CZ-625 00 Brno, Czech Republic. E-mail: svacinova@med.muni.cz

\section{Introduction}

Arterial compliance is a property of the arterial wall enabling a dampening of the amplitude pulse wave. Compliance is defined as a volume difference induced by the pressure difference. Elastance is a reciprocal value of compliance and it decreases with age. A low compliance is frequently a marker of endothelial dysfunction and of changes in the structure of the arterial wall and results in different pathological states. Most complications are associated with the metabolic syndrome (Neutel et al. 1992).

Arterial compliance becomes impaired at an early stage of hypertension when arterial pressure may be only slightly elevated (Ventura et al. 1984). A low compliance of great arteries as a sign of angiopathy is one of the predictors of cardiovascular risks like an ischemic heart disease, myocardial infarction, or stroke.

The methods of arterial compliance measurement are direct and indirect. Direct methods are based on pulsatile pressure measurement (taken invasively or non-invasively) and pulsatile diameter changes within the artery. The pulsatile diameter of great arteries could be obtained by angiography, echocardiography, magnetic resonance, and an intravascular wall motion detector. The compliance of middle or small arteries was measured by Doppler velocimetry, two-dimensional ultrasound or plethysmography. Intravascular ultrasound is a method 
assessing pulmonary, aortic, coronary and brachial arterial compliance (Nakatani et al. 1995).

Compliance can also be evaluated from the arterial pulse-wave contours obtained intra-arterially together with hemodynamic estimates (Neutel et al. 1992).

Indirect methods reflect non-invasive estimations of the compliance conditions (but not absolute values) of great arteries. Compliance can be estimated by pulse-wave velocity, non-invasive pulsewave contour analysis (augmentation index, Buckberg index, spectral analysis) (Yasmin and Brown 1999), by Fourier analysis of pressure and flow waveforms, or by the stroke volume/pulse pressure ratio. Structural changes of the arterial wall (mainly aorta and carotids) are visible and measurable by ultrasound as a higher intima-media thickness.

In the last ten years researchers focused on the study of the arterial pressure-volume relationship and the corresponding arterial compliance by photoplethysmographic methods. Finger photoplethysmography is a measurement technique enabling detection of arterial blood volume changes in the finger in relationship to pressure changes in the finger arteries. The finger cuff contains two optoelectronic components: an infrared light source to illuminate the tissue and a photodetector for the measurement of the small variation in light intensity associated with changes in perfusion of finger vessels. The principle of photoplethysmography is used also in continuous blood pressure measurement according to Penaz (e.g. Finapres).

Previously, we developed a new method for studying the viscoelastic properties of finger arteries and we used for this method a term vibration plethysmography (Penaz et al. 1997). In principle, relatively fast vibrations $(50 \mathrm{~Hz})$ were superimposed on a slowly changing pressure in the finger cuff and the evoked oscillation changes in vascular volume were measured by a photo-electric sensor as the vibration plethysmogram. This signal was split into two components: the normal photo-electric plethysmogram obtained by filtering out the fast components, and the waveform of dynamic vascular compliance. The continuous blood pressure was measured on an adjacent finger (Finapres 2300). Both cuffs were necessary for transmural pressure computation from differences between blood pressure and applied pressure. The shape and polarity of the dynamic vascular compliance waveform depended upon transmural pressure (blood pressure - cuff pressure). It was biphasic with maximal value at transmural pressure slightly lower than mean blood pressure, whereas the normal photo-electric plethysmogram was nearly proportional to the transmural pressure.

Similar techniques, i.e. combination of two photoplethysmographic finger cuffs are used in the last decade: One cuff is used for a continual blood pressure measurement from one finger. The other cuff on the adjacent finger is used for estimation of the relationship between the amplitude of volume changes and the pressure changes applied in the cuff expressed as transmural pressure changes (Talts et al. 2006). The estimated values are substituted as parameters in the mathematical pressure-volume model based, for example, on an exponential function (Tanaka et al. 2011) or an asymmetric arctangent function (Talts et al. 2006).

The aim of this study was to develop a new modification of a vibration plethysmographic method of an arterial compliance analysis in dependence on the transmural pressure. Finally, we analyzed the compliance of a group of volunteers.

\section{Methods}

Theoretical background of the dynamic pressure-volume relationship

In summary, the pressures acting on the arterial wall are as follows: blood pressure $B P(t)$ is acting on the inner side of the arterial wall, the applied cuff pressure $P_{c}(t)$ is acting on the outer side of the arterial wall, and the transmural pressure $P_{t}(t)$ is the resultant pressure originating from the elastance of the arterial wall in dependence on volume. The pressures are time-varying, therefore we have to consider pressure $P_{d}(t)$ given by dissipative forces (which act against the moving mass in the viscose environment, mainly against blood flow) and pressure $P_{i}(t)$ given by inertial forces (caused by acceleration of the moving mass).

From the physical analysis of the problem, we derive the equation for the dynamic equilibrium of pressures acting on the arterial wall during its movement in radial direction, and hence for the volume changes during time. This equation expresses pressures $P_{i}, P_{d}$ and $P_{t}$ as functions of the volume $v(t)$ and derivatives of $v(t)$.

$B P(t)-P_{c}(t)=P_{i}\left[v(t), \frac{d^{2}}{d t^{2}} v(t)\right]+P_{d}\left[v(t), \frac{d}{d t} v(t)\right]+P_{t}[v(t)]$ 


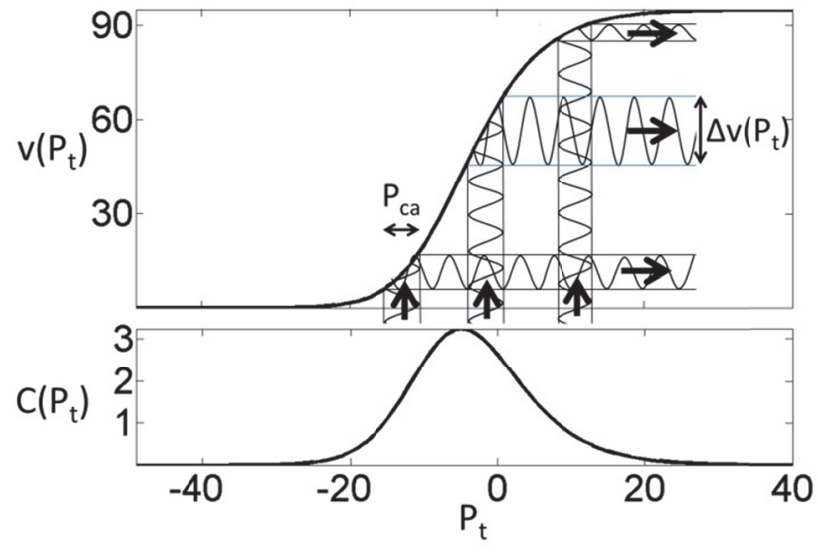

Fig. 1. Above: curve of volume $v\left(P_{t}\right)$ at transmural pressure $P_{t}$. There is an illustration of a sinusoidal pressure vibrations at different $P_{t}$ and constant amplitude $P_{c a}$ applied by the finger cuff and a response to this pressure vibrations in the form of the arterial volume vibrations at $\Delta v\left(P_{t}\right)$. Below: compliance $C\left(P_{t}\right)$ as a derivation of the volume-pressure curve $v\left(P_{t}\right)$.

It is known from many observations that the dependence of volume of unitary arterial length $v$ on transmural pressure $P_{t}$ is non-linear. This dependence can be expressed by an asymmetric sigmoid often approximated by the function:

$v\left(P_{t}\right)=A /\left(\left(1+e^{-a\left(P_{t}-P_{o}\right)}\right) \cdot\left(1+e^{-b\left(P_{t}-P_{o}\right)}\right)\right)$ where $A$ is the maximal volume (asymptote of function $v(P t))$. Coefficients $a$ and $b$ describe asymmetry of function (2). $P_{o}$ defines the shift function relative to the start axis $P_{t}$. The derivation of volume $v\left(P_{t}\right)$

$\frac{d}{d P_{t}} v\left(P_{t}\right)=C\left(P_{t}\right)=1 / E\left(P_{t}\right)$

is the compliance $C\left(P_{t}\right)$ and the reciprocal value $E\left(P_{t}\right)$ is the elastance (Fig. 1).

To compute the frequency characteristic of the pressure-volume relationship by the application of Fourier transform on volume $v(t)$ and pressure $P_{c}(t)$, we have to linearize equation (2) by a Taylor polynomial. By this approach we get the frequency response in analytical form in terms of

$\mathcal{F}[v(t)] / \mathcal{F}\left[P_{c}(t)\right]$

for a steady-state oscillation excited by the harmonic vibration pressure $P_{c a} \cdot \sin (2 \pi f . t)$ with a "small" amplitude $P_{c a}$ around the point $\left[P_{t 0}, v_{0}\right]$ of function (2). Values $P_{t 0}$ are given by the adjustable constant pressure values $P_{c s s}$ in the finger cuff (Fig. 2).
A

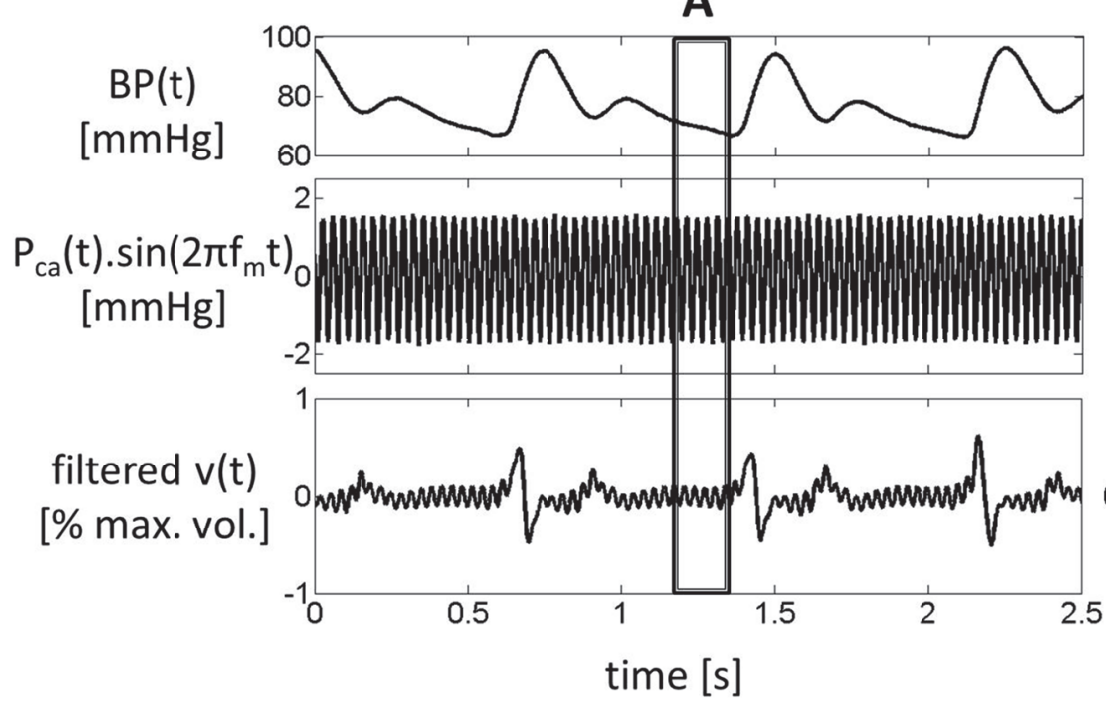

B

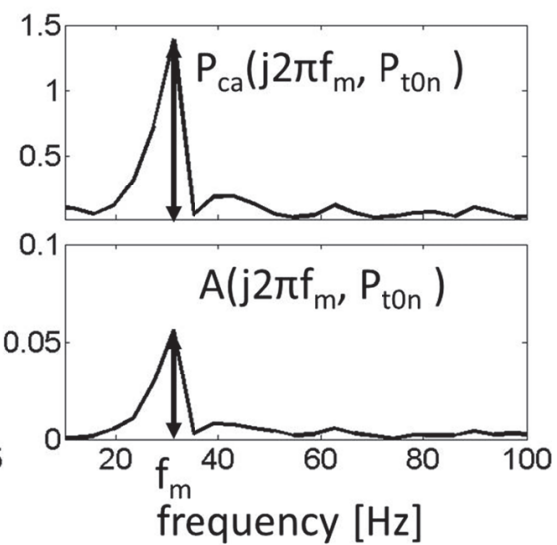

Fig. 2. A (left): an example of $4 \mathrm{~s}$ lasting recordings. $B P(t)$ : blood pressure, $P_{c a}(t) \cdot \sin \left(2 n f_{m} t\right)$ : cuff pressure vibration, filtered $v(t)$ : volume vibration obtained by photodiode (filtered signal). In the frame is an example of one end-diastolic part of the analyzed curves suitable for spectral analysis. B (right) above: spectrum of applied pressure vibration; $P_{c a}\left(j 2 n f_{m}, P_{t 0 n}\right)$ is an amplitude of applied pressure vibration. B (right) below: spectrum of volume vibration $v(t)$, where $A\left(j 2 n f_{m}, P_{t o n}\right)$ is an amplitude of arterial volume vibration. Frequency $\mathrm{f}_{\mathrm{m}}$ : an example at one vibration frequency. (These amplitudes at different frequencies and transmural pressures serve to a frequency characteristic computation; see in the text.) 
The conditions of the steady oscillations mentioned above are relatively well fulfilled for the end of the diastolic pulse curve: for $t$ from the time-interval $I=\left(t_{d}-T_{p / 4}, t_{d}\right)$, where $t_{d}$ is a moment of the enddiastolic pressure, and $T_{p / 4}$ is a quarter of the pulse interval. In this interval, blood pressure changes are the smallest. The duration of $I$ is sufficient for the application of harmonic analysis for the lowest test frequency of pressure vibration $(20 \mathrm{~Hz})$, as documented in Figure 2.

Direct components on both sides of the equation of a dynamic balance of pressures are equal within the case of a small volume amplitude $\Delta v(t)$ around a point $\left[P_{t 0}, v_{0}\right]$.

Expressing the pressure vibration and the corresponding volume vibration in complex form, we get the frequency characteristic of the vibration transmitted by the arterial wall:

$$
\begin{aligned}
F\left(j 2 \pi f, P_{t 0}\right) & =\left(A\left(j 2 \pi f, P_{t 0}\right)\right) / P_{c a}\left(P_{t 0}\right)= \\
& =1 /\left(E\left(P_{t 0}\right)-(2 \pi f)^{2} K_{m}+j 2 \pi f K_{d}\left(P_{t 0}\right)\right)
\end{aligned}
$$

The modulus of $F\left(j 2 \pi f, P_{t o n}\right)$ can be expressed as a network of the amplitude frequency characteristics

$$
\left|F\left(j 2 \pi f_{m}, P_{t 0 n}\right)\right|=\left|A\left(j 2 \pi f_{m}, P_{t 0 n}\right)\right| /\left|P_{c a}\left(j 2 \pi f_{m}, P_{t 0 n}\right)\right|
$$

for a vector of frequencies $\left[f_{m}\right], m=1,2, \ldots, M$ of the pressure vibrations transmitted and a vector of transmural pressures $\left[P_{t 0 n}\right], n=1,2, \ldots, N$. The amplitudes $A\left(j 2 \pi f_{m}\right.$, $\left.P_{t 0 n}\right)$ are obtained by a fast Fourier transform (FFT) on the observed volume vibrations $\Delta v(t) . P_{c a}$ is slightly influenced by the transmural pressure, therefore the amplitudes $P_{c a}\left(j 2 \pi f_{m}, P_{t o n}\right)$ are obtained by an FFT of the $P_{c a}\left(P_{t 0}\right) \cdot \sin (2 \pi f t)$ (Fig. 2).

The compliance

$\mathrm{C}\left(\mathrm{P}_{\mathrm{ton}}\right)=1 / \mathrm{E}\left(\mathrm{P}_{\mathrm{ton}}\right)$

is a static feature of the arterial wall and can be considered as a frequency characteristic $\left|F\left(j 2 \pi f_{m}, P_{t 0 n}\right)\right|$ at frequencies $f=0$. A reason for this consists in the fact that the amplitudes of transmitted vibrations decrease with increasing vibratory frequency. This is caused by the inertial forces of the moving tissues. To compute $C\left(P_{t 0}\right)$, we use a real part of the reciprocal form of a frequency characteristic (5) as a regression function with a vector $\left[f_{m}\right]$ as an argument of the predictor

$\operatorname{Re}\left[F^{-1}\left(j 2 \pi f_{m}, P_{t 0 n}\right)\right]=E\left(P_{t 0 n}\right)-\left(2 \pi f_{m}\right)^{2} \cdot K_{m}$
The results are the value of the elastance $E\left(P_{t o n}\right)$ and the compliance $C\left(P_{t o n}\right)$ as a reciprocal value of $E\left(P_{t 0 n}\right)$.

\section{Measurement procedures}

Measurement conditions and finger cuff setting

The resting measurement was carried out at room temperature $\left(24^{\circ} \mathrm{C}\right)$, in sitting position without movements and at a state of the subject when the fingers were not cold. The volunteers did not take any vasoactive drugs before the measurement. Firstly, values of systolic and diastolic blood pressure were taken by the classic oscillometric method. Then, the finger cuff for continual blood pressure measurement (Portapres) was placed on the middle phalanx of the fourth finger. The occlusion finger cuff for the application of pressure vibration was placed on the middle phalanx of the third finger.

\section{Normalization of the arterial volume}

The especially modified occlusion finger cuff was equipped with a source of infrared light and a photoelectric sensor for the measurement of arterial volume. The absorption of infrared light by the finger tissue was dependent on arterial volume. The finger cuff was inflated on a constant pressure $P_{c s s}$ of $30 \mathrm{~mm} \mathrm{Hg}$ (without vibrations) and the photoelectric signal was measured. This value was considered as $100 \%$ of the maximal arterial volume. After that, the finger cuff was inflated on a pressure $P_{c s s}$ of $170 \mathrm{~mm} \mathrm{Hg}$. The arteries were closed at this pressure. The light passing through the tissue at a pressure of $170 \mathrm{~mm} \mathrm{Hg}$ was considered as $0 \%$ of the maximal arterial volume. By this approach the arterial volume could be indicated in units of $\%$ of the maximal volume. As a result we obtain frequency characteristics normalized to the arterial volume.

\section{Measurement protocol}

The finger cuff operated on the artery at a pressure $P_{c}(t)=P_{c s s}+P_{c a} \cdot \sin (2 \pi f t)$. The pressure sequentially took 10 values $P_{c s s}$ from 170 to $30 \mathrm{~mm} \mathrm{Hg}$. The pressure vibrations $P_{c a} \cdot \sin (2 \pi f t)$ of a constant amplitude $P_{c a}=2 \mathrm{mmHg}$ and frequencies $\mathrm{f}=(20,25,30$, $35,40) \mathrm{Hz}$ were sequentially superimposed to each pressure value $P_{c s s}$. Thus we used 5 x 10 combinations of $P_{c}(t)$ settings. Each pressure $P_{c}(t)$ was applied for a time interval lasting 5 seconds. Simultaneously, we recorded the arterial volume vibration $v(t)$ as a response to the pressure vibrations $P_{c}(t)$. Finally, the frequency 
characteristics and compliance in dependence on transmural pressure were evaluated.

\section{Subjects}

The special original equipment described and the especially designed measurement protocol were tested on 6 young healthy normotensive subjects ( 2 women and
4 men) aged $21 \pm 1$ years and two elderly subjects (1 diabetic woman and 1 heallthy man) aged 65 and 70 years; resting blood pressures 130/85 $\mathrm{mm} \mathrm{Hg}$ and 120/80 $\mathrm{mm} \mathrm{Hg}$.

The Ethics Committee of Masaryk University approved the study and all participants of the study gave their consent.
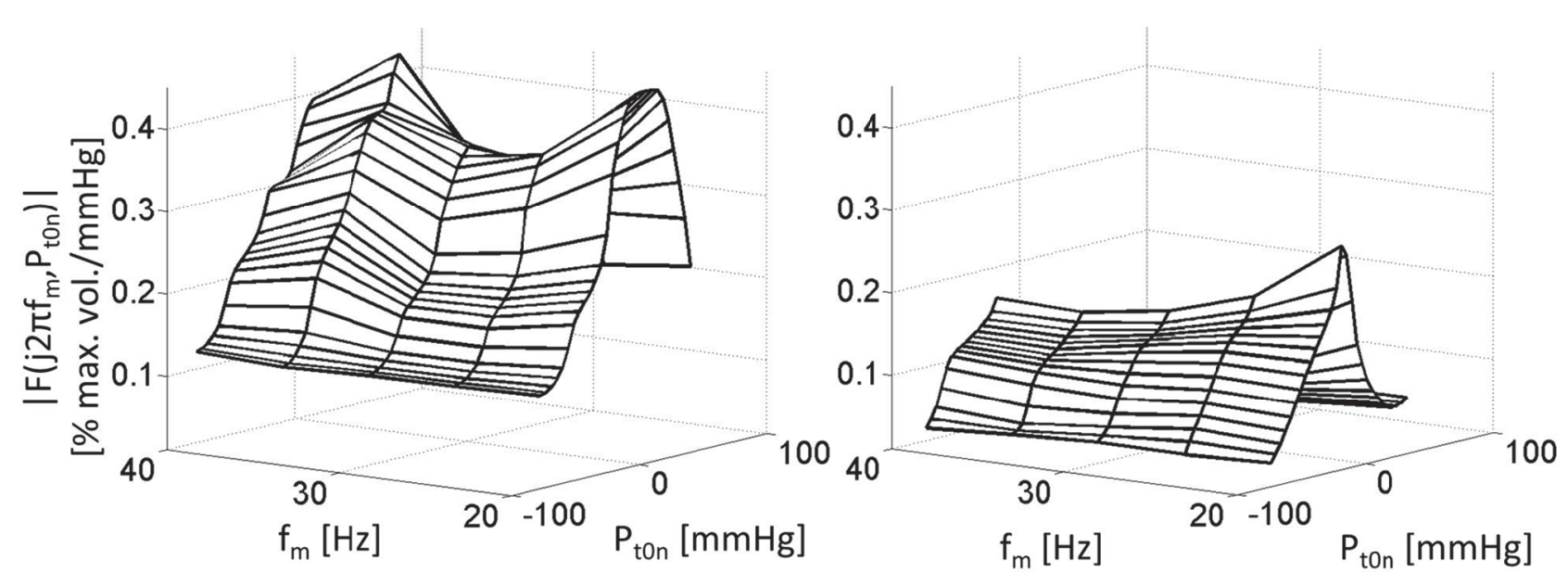

Fig. 3. Network of frequency characteristics $\left|F\left(j 2 n f_{m}, P_{t o n}\right)\right|$ of the pressure vibration transmitted to the volume vibration in dependence on vibration frequency $f_{m}$ and transmural pressure $P_{t 0 n}$. Left: Frequency characteristic network of the 28 years old woman (normotensive, nonsmoker). Right: Frequency characteristic network of the 70 years old man (normotensive, smoker).

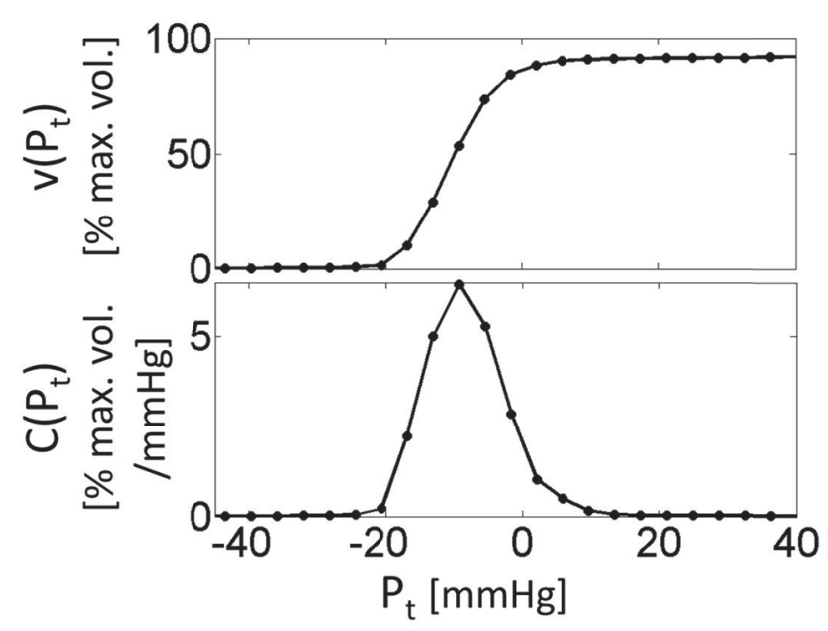

Fig. 4. Above: An example of curve of an arterial volume $v\left(P_{t}\right)$ in dependence on a transmural pressure $\mathrm{P}_{\mathrm{t}}$. Below: Evaluated arterial compliance $\mathrm{C}\left(\mathrm{P}_{\mathrm{t}}\right)$ in dependence on a transmural pressure $P_{\mathrm{t}}$.

\section{Results}

The resulting frequency characteristics of arterial wall responses to the applied pressure vibrations differed between the subjects. An example of three-dimensional graphs of the network of the frequency characteristic $\mid F\left(j 2 \pi f_{m}, P_{t o n} \mid\right.$ of the arterial wall is shown in Figure 3.
This frequency characteristic provides information about the ability of the arterial wall to transmit vibrations of different frequencies from the pressure in the cuff to the arterial volume in dependence on transmural pressure. This graph demonstrated that an increased pressure vibratory frequency caused a decrease of the transmission amplitude. This effect could be influenced by arterial compliance. On the example of frequency characteristics in Figure 3 it appeared that the frequency characteristics of an older individual had lower values, especially at a higher frequency, compared to the frequency characteristic of a young individual.

An arterial compliance evaluated by regression analysis from the frequency characteristic is presented in Figure 4 . The values of the static compliance were often much higher (1-6\% maximal volume $/ \mathrm{mm} \mathrm{Hg}$ ) than those of the frequency characteristic $(0-1 \%$ maximal volume $/ \mathrm{mm} \mathrm{Hg}$ ). The low values of the dynamic compliance (frequency characteristic) were given by the fact that most of the power of the transmitted vibrations was lost due to dissipative and inertial forces. The higher values of the static compliance were caused by the regression operation of the real part of the reciprocal values of the frequency characteristics. The compliance $\mathrm{C}\left(\mathrm{P}_{\mathrm{t}}\right)$ of six healthy normotensive young subjects is 
presented in Figure 5. Numeric integration of the arterial compliance provides a curve of the arterial volume in dependence on transmural pressure.

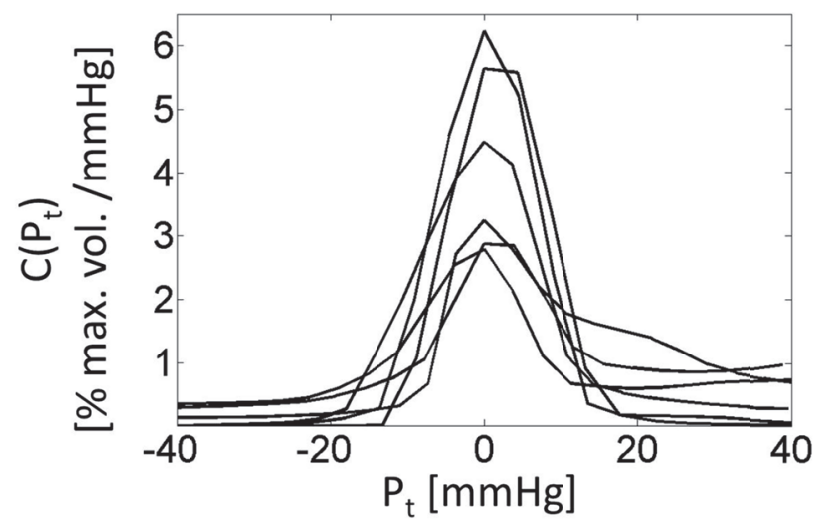

Fig. 5. An example of compliance $C\left(P_{t}\right)$ of six healthy normotensive young subjects. Graphs are aligned to the pressure value of the occurrence of the maximal value of the peak, therefore slight differences in the individual pressures were adjusted to 0 .

\section{Discussion}

We have developed a new original method of the determination of dynamic compliance of small arteries, which could serve as a new way to examine small arteries under different physiologic and pathologic states (hypertension, diabetes mellitus, impaired control of finger blood flow in special disorders). Our preliminary results are presented in this paper.

Some studies estimated arterial compliance based on finger photoplethysmography using pressurevolume changes at changeable transmural pressures (Talts et al. 2006). Our study added additional information on the ability of the arterial wall to transmit vibrations of different frequencies from the applied pressure to arterial volume vibrations by a different gain. The change of the dynamic compliance (frequency characteristic) with the frequency of vibrations may not be monotonic. This is due to the achievement of the resonant frequency of the arterial wall at a given transmural pressure and the frequency of vibrations. This resonant frequency is determined by the elasticity (directly) and the mass (indirectly) of the artery. The amplitude of the resonance depends on the resistance of the blood flow (indirectly). All reciprocal values of the frequency characteristic are included into the regression analysis for computing the appropriate parameter - static elastance. Compliance is the reciprocal value of the elastance competent for the frequency characteristic. We showed that arterial compliance influenced the shape of the frequency characteristic of the arterial wall. Lower compliance seems to be linked with a decrease of the amplitude of transmitted vibrations at higher frequencies.

The shape of the estimated compliance at changeable transmural pressure was asymmetric in agreement with the results described by other researchers (Talts et al. 2006). Most studies evaluated compliance by estimating blood-volume parameters of the artery fitted into a parametric model (Penaz et al. 1997, Talts et al. 2006, Tanaka et al. 2011). We were able to evaluate compliance by this model, but also directly for each value of transmural pressure.

We observed individual variability of the results: a shift of the compliance curve peak toward negative transmural pressure in the range from $0 \mathrm{~mm} \mathrm{Hg}$ to $-20 \mathrm{~mm} \mathrm{Hg}$, a slope of the volume-pressure curve and asymmetry of the compliance curve, similarly as Penaz et al. (1997), Talts et al. (2006). These observations could be given by an arterial compliance but also could be influenced by the above mentioned physical factors such as viscoelasticity and mass of the finger, artery and blood. A shift of the compliance curve peak toward negative transmural pressure was probably given by the shape of the arterial wall. The transmural pressure of the compliance curve peak was determined by the maximal amplitude of the wall vibration, which was the greatest in negative transmural pressure due to the rounded arterial wall. Furthermore, the shift of the compliance curve peak could be caused by a property of non-invasive oscillometric finger blood pressure estimation: blood pressure pulse amplitude and shape affect the results of a diastolic and systolic blood pressure measurement (Raamat et al. 1999). This was the reason for our extensive theoretical physical background in which we described parameters determining the forces affecting the volume response to pressure vibrations as inertial forces and dissipative forces. This background can be helpful for further research of compliance estimated by the frequency characteristic of the arterial wall.

\section{Conflict of Interest}

There is no conflict of interest.

\section{Acknowledgements}

This study was supported by MUNI/A/0957/2013 and European Regional Development Fund - Project FNUSA-ICRC (No. CZ.1.05/1.1.00/02.0123). 


\section{References}

CHANG S, WANG JJ, SU HM, LIU CP: On calculating the time-varying elastance curve of a radial artery using a miniature vibration method. In: 13th International Conference on Biomedical Engineering (ICBME) 2008. Vol. 23. Springer, Berlin - Heidelberg, 2009, pp 540-542.

NAKATANI S, YAMAGISHI M, TAMAI J, GOTO Y, UMENO T, KAWAGUCHI A, YUTANI C, MIYATAKE K: Assessment of coronary artery distensibility by intravascular ultrasound. Application of simultaneous measurements of luminal area and pressure. Circulation 91: 2904-2910, 1995.

NEUTEL JM, SMITH DHG, GRAETTINGER WF, WEBER MA: Dependency of arterial compliance on circulating neuroendocrine and metabolic factors in normal subjects. Am J Cardiol 69: 1340-1344, 1992.

PENAZ J, HONZIKOVA N, JURAK P: Vibration plethysmography: a method for studying the visco-elastic properties of finger arteries. Med Biol Eng Comput 35: 633-637, 1997.

RAAMAT R, TALTS J, JAGOMAGI K, LANSIMIES E: Mathematical modelling of non-invasive oscillometric finger mean blood pressure measurement by maximum oscillation criterion. Med Biol Eng Comput 37: 784-788, 1999.

TALTS J, RAAMAT R, JAGOMAGI K: Asymmetric time-dependent model for the dynamic finger arterial pressurevolume relationship. Med Biol Eng Comput 44: 829-834, 2006.

TANAKA G, YAMAKOSHI K, SAWADA Y, MATSUMURA K, MAEDA K, KATO Y, HORIGUCHI M, OHGURO H: A novel photoplethysmography technique to derive normalized arterial stiffness as a blood pressure independent measure in the finger vascular bed. Physiol Meas 32: 1869-1883, 2011.

VENTURA H, MESSERLI FH, OIGMAN W, SUAREZ DH, DRESLINSKI GR, DUNN FG, FREISIN E, FROHLICH ED: Impaired systemic arterial compliance in borderline hypertension. Am Heart J 108: 132-136, 1984.

YASMIN, BROWN MJ: Similarities and differences between augmentation index and pulse wave velocity in the assessment of arterial stiffness. Quart J Med 92: 595-600, 1999. 